\title{
Portable biosensor for chronic malaria detection
}

\begin{abstract}
Malaria is a leading parasitic disease endangering the lives of half of the world's population. Microfluidics paves way to indispensable portable sensors to enable early sensing hence defend lives. In this paper, we demonstrate a portable sensor based on dielectric spectroscopy capable of pre-diagnostic and malaria parasite detection. The complete sensor system consists of the separation zone to separate the white blood cells and the detection zone, which is connected to portable impedance circuit board to measure the dielectric value of red blood cells to detect the infection. The current work highly emphasize on the separation part only while a little light is shed on detection zone.
\end{abstract}

Keyword: Dielectric spectroscopy; Malaria detection; Microweir separation 